\title{
Contribution of the Charge Image Potential to Carrier Confinement in Graded Si-Based Quantum Wells
}

\author{
T. A. S. Pereira, E. W. S. Caetano, J. A. K. Freire, V. N. Freire, and G. A. Farias \\ Departamento de Física, Universidade Federal do Ceará, Centro de Ciências Exatas \\ Campus do Pici, Caixa Postal 6030, 60455-900 Fortaleza, Ceará, Brazil
}

Received on 31 March, 2003

\begin{abstract}
In order to analyze the charging effects on electronic spectrum of Silicon ( $\mathrm{Si}$ ) based quantum wells (QW's), we use a method based on the calculation of the image charge potential by solving Poisson equation in cylindrical coordinates. The numerical results shows that the confined electron ground state energy level can be shifted by more than $50 \mathrm{meV}$ due to the contribution of the image charges to the confinement potential of the graded quantum well.
\end{abstract}

\section{Introduction}

The contribution of the image charge to the potential structure of $\mathrm{Si} / \mathrm{SiO}_{2}$ heterojunctions have been widely studied in the past [1]. At that time, however, the devices dimensions were above the $\mu \mathrm{m}$ range, and in this case effects of roughness were very limited. With the shrinkage of the device dimensions to the nanoscale, the effect of the image potential on electron transmission and electric current in the direct tunnelling regime of ultra-thin MOS structures became a matter of interest [2], which is driving new efforts for the understanding of the image potential contribution to nanoelectronics [3]. In particular, the recent search for the $\mathrm{SiO}_{2}$ replacement by high- $k$ dielectrics will point the necessity for a better description of the dielectric mismatch in semiconductor nanostructures, which also must take into account graded interface effects.

The purpose of this work is to present results on the calculation of the charge image potential contribution to the carriers' confinement in graded Si-based quantum wells $\left(\mathrm{Si} / \mathrm{SiO}_{x}\right)$. A linear variation of the dielectric constant through the graded quantum well interface is assumed, which allows to avoid the sharp interface-related divergence in the image potential contribution. Our model is based on the work proposed by Stern [4] and extended for 2D quantum well structures. This model ignores the atomic nature of the materials and also neglects the mobility of the carries, which can modify the image potential in the interface between an insulator and a semiconductor. Despite these assumptions, our approach is consistent with the approach of the effective mass, which has been used commonly to describe electrons near interfaces of films [5].

\section{Image Potential}

The electrostatic potential $\phi(r)$ due to the presence of a point charge $Q$ located at $r_{0}$ in a medium with a spatially varying permittivity $\varepsilon$ is the solution of Poissons equation

$$
\nabla \cdot \varepsilon(z) \nabla \phi=-Q \delta\left(\vec{r}-\vec{r}_{0}\right)
$$

Since $\varepsilon$ only depends on $z$ coordinate, we have the freedom to choose the origin of the $x y$ plane at the position of the point charge $Q$. We can define a radial coordinate $R$, and the potential $\phi(r)$ can be expanded in a Fourier-Bessel form [4]:

$$
\phi(\vec{r})=\int_{0}^{\infty} q J_{0}(q R) A_{q}(z) d q
$$

where $J_{0}(x)$ is the Bessel function of order zero. The coefficients $A_{q}(z)$ satisfies the following equation

$$
A_{q}^{\prime \prime}(z)-q^{2} A_{q}(z)+\frac{\varepsilon^{\prime}(z)}{\varepsilon(z)} A_{q}^{\prime}(z)=-\frac{Q \delta\left(z-z_{0}\right)}{2 \pi \varepsilon\left(z_{0}\right)},
$$

with boundary conditions $A_{q}(z) \rightarrow 0$ when $|z| \rightarrow 0$. The solution of the above equation can be expressed as

$$
A_{q}(z)=\alpha I_{0}(\eta)+\beta K_{0}(\eta)
$$

where $\alpha$ and $\beta$ are coefficients related with the boundary conditions, and $\eta$ is a function of $z$, the interface thickness and the dielectric constant $[4,6]$.

In the case of a dielectric constant independent of $z$, the solution of Eq. (3) is

$$
A_{q}^{0}(z)=\frac{Q}{4 \pi \varepsilon_{\left(z_{0}\right)}} e^{-q\left|z-z_{0}\right|} .
$$

The image charge potential equation including the effect of the spatial variation of the dielectric constant on the point charge $Q$ can be expressed as

$$
V^{i m}(z)=\frac{1}{2} \int_{0}^{\infty} q\left[A_{q}(z)-A_{q}^{0}(z)\right] d q .
$$




\section{Modelling $\mathrm{Si} / \mathrm{SiO}_{x}$ QW's}

The carrier Hamiltonian in non-abrupt $\mathrm{Si} / \mathrm{SiO}_{x}$ single QWs can be written, within the effective mass approximation, in the following way:

$$
H_{i}\left(z_{i}\right)=-\frac{\hbar^{2}}{2} \frac{\partial}{\partial z_{i}}\left[\frac{1}{m_{i}\left(z_{i}\right)} \frac{\partial}{\partial z_{i}}\right]+V_{i}\left(z_{i}\right)+V_{i}^{i m}\left(z_{i}\right)
$$

where $z$ is the QW growth direction, $m_{i}\left(z_{i}\right)$ and $V_{i}\left(z_{i}\right)$ is the carrier perpendicular effective mass and effective potential, respectively, with $i=(e, l h, h h)$, and $V_{i}^{i m}\left(z_{i}\right)$ is the image charge contribution (see Eq. (6)).

To describe the interface, we use the model of Freire $e t$ al. [7], where $\varepsilon_{i}\left(z_{i}\right), m_{i}\left(z_{i}\right)$ and $V_{i}\left(z_{i}\right)$ are dependent on the interfacial aluminum molar fraction $\chi(z)$ in the structure, which is a linear function of the growth coordinate $z$.

$$
\begin{aligned}
\varepsilon_{i}\left(z_{i}\right) & =\varepsilon_{a}+\varepsilon_{b} \chi(z), \\
m_{i}\left(z_{i}\right) & =\mu_{1}+\mu_{2} \chi(z), \\
V_{i}\left(z_{i}\right) & =v_{a}+v_{b} \chi(z) .
\end{aligned}
$$

In Fig. 1(a) we have the image of a $\mathrm{Si} / \mathrm{SiO}_{2}$ film showing the existence of a non-abrupt transition region between the two materials, while a sketch of the dielectric function through the QW structure is shown in Fig. 1(b).
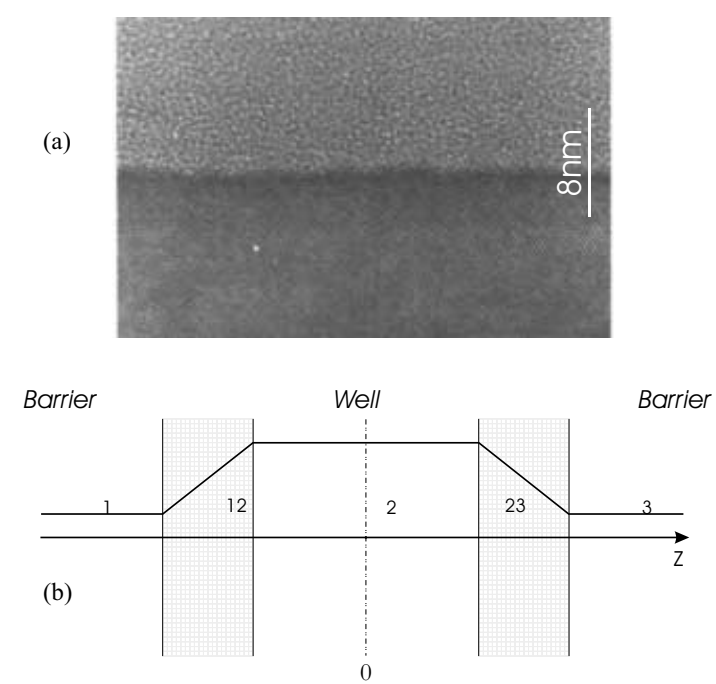

Figure 1. (a) Interface showing the non-abrupt interface of a $\mathrm{Si} / \mathrm{SiO}_{2}$ film [8]. (b) Schematic diagram of the dielectric quantumwell structure.

Using the above considerations, we have the following Schrödinger equation for the perpendicular motion:

$$
\left[H_{i}\left(z_{i}\right)-E_{i}\right] \psi_{i}\left(z_{i}\right)=0 .
$$

The eigenvalues and eigenfunctions of the above wave equation are calculated through a matrix transfer scheme [7].

\section{Carrier Energy}

We calculated the carrier energy in $\mathrm{Si} / \mathrm{SiO}_{x}$ single quantum wells including the charge image effects. The parameters (energy gap, dielectric constant, and effective masses) used for $\mathrm{Si}$ and $\mathrm{SiO}_{2}$ are presented in Table I. The $\mathrm{SiO}_{x}$ parameters are obtained through linear interpolation.

Table I. Si[9] and $\mathrm{SiO}_{2}$ [10] parameters

\begin{tabular}{ccc}
\hline Parameters & $\mathrm{Si}$ & $\mathrm{SiO}_{2}$ \\
\hline$E g(e V)$ & 1.05 & 8.9 \\
$\varepsilon / \varepsilon_{0}$ & 11.7 & 2.1 \\
$m_{e}^{\perp} / m_{0}$ & 0.173 & 0.3 \\
$m_{h h}^{\perp} / m_{0}$ & 0.533 & 5.0 \\
\hline
\end{tabular}

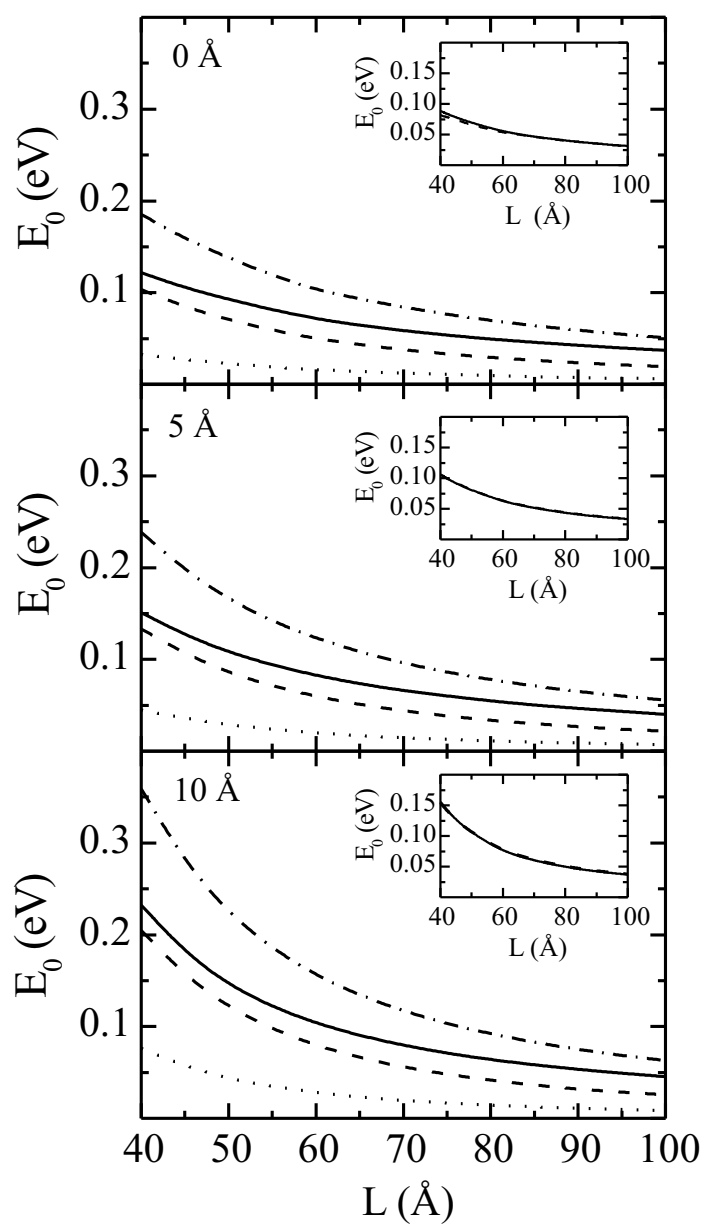

Figure 2. Carrier energy as a function of the well width, without the image charge effects $e^{-}$(dashed) and $h h$ (dotted) and with the image charge effects $e^{-}$(dotted-dashed) and $h h$ (solid). In the inset, the difference between the carrier energy, $e^{-}$(dashed) and $h h$ (solid), with and without the image charge effects.

In the present calculation two effects are considered, that is, the charge image potential and the graded interface. Fig. 2 shows the electron (dashed and dotted-dashed) and heavy hole (dotted and solid) energy as a function of the quantum well width, for interfaces thicknesses of 0,5 and $10 \AA$. The numerical results presented in the solid and dotted-dashed curves takes into account the image charge effects decribed by the last term in the Hamiltonian of Eq. (7). These effects are not included in the calculations of the dotted and dashed curves. 
In the abrupt interface picture, the effects observed are restricted to the image charge. As shown in Fig. 2 (top) the ground state energies for electrons and heavy holes increases when the charge image potential is considered. Since the $\mathrm{SiO}_{2}$ dielectric constant is higher than $\mathrm{Si}$, the image charge has the same signal as the charge inside the quantum well. As a result, the charge image potential increases the carries confinement inside the quantum well and consequently the ground state energies of the carriers. In the inset we observe that the energy increasing does not differ significatively for electrons and heavy holes.

Considering non-abrupt interfaces with thicknesses 5 and $10 \AA$, Fig. 2 (middle and bottom, respectively) shows the results with and without the charge image potential. Since the graded interface model considered here decreases the effective quantum well width, we observe that the ground state energies of the carriers increases with the interface thickness. As can be seen, the two effects, charge image potential and non-abrupt interface, can increase the ground state energy of the carries by more than $100 \mathrm{meV}$.

In conclusion, we observed that the effects of the charge image potential and the existence of non-abrupt interfaces are important in the ground state energies of quantum well composed by materials with dielectric constants that differ significatively.

\section{Acknowledgments}

This research was supported by the Brazilian Ministry of Culture (MEC-CAPES), the Funding Agency of the Ceará
State in Brazil (FUNCAP), and by $\mathrm{CNPq} / \mathrm{NanoSemiMat}$ grant N. 550.015/01-9.

\section{References}

[1] T Ando A. B. Fowler, and F. Stern, Rev. Mod. Phys. 54, 437 (1982).

[2] L. F. Mao, C. H. Tan, and M. Z. Xu, Microelectronics Reliability 42, 991 (2002).

[3] E. A. Muljarov, E. A. Zhukov, V. S. Dneprovskii, and Yasuaki Masumoto, Phys. Rev. B 62, 7420 (2000); G. Palasantzas, J. Appl. Phys. 82, 351 (1997).

[4] F. Stern, Phys. Rev. B 17, 5009 (1978); ibid., Solid State Commun. 25, 163 (1977).

[5] M. Kumagai and T. Takagahara, Phys. Rev. B 40, 12359 (1989).

[6] T. A. Pereira, J. A. K. Freire, V. N. Freire, and G. A. Farias, Microelectronics Journal 34, 507 (2003).

[7] J. A. K. Freire, G. A. Farias, and V. N. Freire, Solid State Commun. 106, 559 (1998).

[8] Chung Yi, Hyo Uk Kim, Shi Woo Rhee, Sang Ho Oh, and Chan-Gyung Park, J. Vac. Sci. Technol. B 19, 2067 (2001).

[9] G. Theodorou, G. Tsegas, P. C. Kelires, E. Kaxiras, Phys. Rev. B 60, 11494 (1999).

[10] J. R. Chelikowsky and M. Schlüter, Phys. Rev. B 15, 4020 (1997); B. Brar, G. D. Wilk, A. C. Seabaugh, Appl. Phys. Lett. 69, 2728 (1996). 